\title{
Estimation of Rates of Recombination and Migration in Populations of Plant Pathogens-A Reply
}

\author{
J. Zhan, C. C. Mundt, and B. A. McDonald
}

First and third authors: Institute of Plant Sciences, Phytopathology Group, ETH Zentrum/LFW, Universitätstrasse 2, CH-8092 Zürich, Switzerland; and second author: Department of Botany and Plant Pathology, Oregon State University, Corvallis. Accepted for publication 3 January 2000.

\begin{abstract}
Zhan, J., Mundt, C. C., and McDonald, B. A. 2000. Estimation of rates of recombination and migration in populations of plant pathogens-A reply. Phytopathology 90:324-326.

We find that the maximum likelihood method proposed by J. K. M. Brown has deficiencies that limit its usefulness for actual data sets. We propose two alternative statistical methods based on maximum likelihood that could be used to quantify rates of recombination and immigration in

fungal populations. We also show that minor modification of our original method, which was based upon posterior probabilities, leads to a result that is identical to one of the maximum likelihood methods. Our previous estimates of the relative contributions of sexual reproduction, asexual reproduction, and immigration to the genetic structure of a Mycosphaerella graminicola population did not change significantly following reanalysis of our data with these new methods.
\end{abstract}

Quantifying the relative contributions of asexual reproduction, immigration, and recombination to the genetic structure of field populations of plant pathogens is very difficult. Recently, Zhan et al. (11) proposed an experimental approach and a mathematical method to estimate the contributions of these three factors to populations of the wheat pathogen Mycosphaerella graminicola. This method was criticized by Brown (1), and he proposed a maximum likelihood approach to make more precise estimates of these parameters. We acknowledge that our original method has some deficiencies, and we realize that maximum likelihood would be a more desirable approach to use when estimating these parameters. In this letter, we would like to build on the maximum likelihood method proposed by Brown (1) and suggest a simpler approach that is more likely to be applicable to conventional data sets.

In his model, Brown (1) assumed that the probability that a genotype would be observed in the mixed population (consisting of a mixture of isolates originating from asexual reproduction, recombination, and immigration) was

$$
p_{g_{i}}^{2}=p_{A} p_{g / A}+p_{M} p_{g / M}+p_{R} p_{g / R}=(1-m-r) p_{g_{i}}^{1}+m M_{g_{i}}+r R_{g_{i}}
$$

in which $p_{g_{i}}{ }^{1}, p_{g_{i}}{ }^{2}, 1-m-r, m$, and $r$ are the probabilities of observing genotype $g_{i}$ in the first collection (parental population) and the second collection (mixed population) and the proportions of the mixed population arising from asexual reproduction, immigration, and recombination, respectively. $M_{g_{i}}$ and $R_{g_{i}}$ are the probabilities for observing genotype $g_{i}$ in the populations of immigrants and recombinants, respectively (note: we used a slightly different notation from Brown [1]). This model assumes that the genetic markers used to differentiate genotypes are neutral, which appears to be true for the restriction fragment length polymorphism (RFLP) markers used in our study (5).

Brown's approach (eq. 3 in literature citation 1) is to determine the joint likelihood of obtaining the genotypes found in all three samples by treating the sample of potential parents (prior collection from

Corresponding author: B. A. McDonald

E-mail address: bruce.mcdonald@ipw.agrl.ethz.ch

Publication no. P-2000-0207-010

(C) 2000 The American Phytopathological Society inoculated plots), the sample of potential immigrants (from uninoculated control plots), and the sample from the mixed population as elements of the same population. Taking the joint likelihood of all three samples has the unfortunate consequence of making an extremely complicated solution that is not likely to be useful in practice. Maximum likelihood with many parameters is problematic, especially if the number of parameters is comparable to the number of observations. Based on Brown's method, the number of parameters to be estimated would be very large, because each genotype is included as a parameter. For a sexual fungus like $M$. graminicola, this could involve thousands of parameters if thousands of genotypes are observed in the mixed population. Another complication is that the three samples are not independent. The number of isolates with genotype $g_{i}$ in the mixed population ( $n_{G 2}$ in Brown's eq. 3 [1]) can be affected by the number of times genotype $g_{i}$ is found in the populations of potential parents $\left(n_{G 1}\right)$ and immigrants $\left(n_{G u}\right)$. This approach is invalid according to the definition of the likelihood function presented by Shenton and Bowman (8) unless a new, conditional term is added, further increasing the complexity. Finally, Brown's (1) formula contains several related parameters, such as $p_{g_{i}}{ }^{1}$ and $R_{g_{i}}\left(V_{G}\right.$ and $R_{G}$ in literature citation 1$) . R_{g_{i}}$ cannot be estimated until all $p_{g_{i}}{ }^{1}$ are known, which is not likely to be the case in an actual data set.

The maximum likelihood approach described by Brown (1) can be simplified considerably in two ways. The first simplification is to consider the genotype frequencies of the mixed population separately from the inoculated and noninoculated populations. This removes the complexity of considering a series of dependent terms. The likelihood that genotype $g_{i}$ will be observed $n_{i}$ times in the mixed population is

$$
L(m, r)=\frac{N !}{n_{1} ! n_{2} ! \ldots n_{i} !} \prod_{i}\left[(1-m-r) p_{g_{i}}^{1}+m M_{g_{i}}+r R_{g_{i}}\right]^{n_{i}}
$$

and the log-likelihood is

$$
\ln L(m, r)=c+\sum_{i} n_{i} \ln \left[(1-m-r) p_{g_{i}}^{1}+m M_{g_{i}}+r R_{g_{i}}\right]
$$

After partial differentiation

$$
\frac{\partial \ln L(m, r)}{\partial m}=\sum_{i} \frac{n_{i}\left(-p_{g_{i}}^{1}+M_{g_{i}}\right)}{(1-m-r) p_{g_{i}}^{1}+m M_{g_{i}}+r R_{g_{i}}}
$$


and

$$
\frac{\partial \ln L(m, r)}{\partial r}=\sum_{i} \frac{n_{i}\left(-p_{g_{i}}^{1}+R_{g_{i}}\right)}{(1-m-r) p_{g_{i}}^{1}+m M_{g_{i}}+r R_{g_{i}}}
$$

The $m$ and $r$ can be estimated by solving equations 4 and 5 simultaneously (8) with mathematical software packages such as Maple V (The Power Edition, student, release 4; Waterloo Maple Inc., Waterloo, Ontario, Canada).

As Brown (1) noted, variances of $m$ and $r$ can be calculated using likelihood methods, though he erred when specifying the asymptotic sampling variances. When sample sizes are large, the variances of the maximum likelihood estimates (MLEs) can be calculated by the term of information (9). The expected information matrix (9) for parameters $m$ and $r$ is

$$
E[I(m, r)]=\left[\begin{array}{ll}
-E\left[\frac{\partial^{2} \ln L(m, r)}{\partial m^{2}}\right] & -E\left[\frac{\partial^{2} \ln L(m, r)}{\partial m \partial r}\right] \\
-E\left[\frac{\partial^{2} \ln L(m, r)}{\partial m \partial r}\right] & -E\left[\frac{\partial^{2} \ln L(m, r)}{\partial r^{2}}\right]
\end{array}\right]
$$

$$
=\left[\begin{array}{c}
-\sum_{i} \frac{n_{i}\left(-p_{g_{i}}^{1}+M_{g_{i}}\right)^{2}}{\left[(1-m-r) p_{g_{i}}^{1}+m M_{g_{i}}+r R_{g_{i}}\right]^{2}}-\sum_{i} \frac{n_{i}\left(-p_{g_{i}}^{1}+M_{g_{i}}\right)\left(-p_{g_{i}}^{1}+R_{g_{i}}\right)}{\left[(1-m-r) p_{g_{i}}^{1}+m M_{g_{i}}+r R_{g_{i}}\right]^{2}} \\
-\sum_{i} \frac{n_{i}\left(-p_{g_{i}}^{1}+M_{g_{i}}\right)\left(-p_{g_{i}}^{1}+R_{g_{i}}\right)}{\left[(1-m-r) p_{g_{i}}^{1}+m M_{g_{i}}+r R_{g_{i}}\right]^{2}}-\sum_{i} \frac{n_{i}\left(-p_{g_{i}}^{1}+R_{g_{i}}\right)^{2}}{\left[(1-m-r) p_{g_{i}}^{1}+m M_{g_{i}}+r R_{g_{i}}\right]^{2}}
\end{array}\right]
$$

Inverting this matrix gives us the large-sample variances of $m$ and $r$ and their covariances.

$$
\{E[I(m, r)]\}^{-1}=\left[\begin{array}{cc}
\operatorname{Var}(m) & \operatorname{Cov}(m, r) \\
\operatorname{Cov}(r, m) & \operatorname{Var}(r)
\end{array}\right]
$$

The hypothesis that the estimated values of $m$ and $r$ differ from arbitrarily chosen values of $m$ and $r$ can be evaluated with a chisquared test using the following formula (7).

$$
\chi^{2} \approx 2\left[\ln L(\hat{m}, \hat{r})-\ln L\left(m_{0}, r_{0}\right)\right]
$$

The chi-squared value can be calculated by substituting into equation 3 the estimated values of $m$ and $r$ and the hypothesized values for $m$ and $r$. This chi-squared test has two degrees of freedom.

This modified maximum likelihood model, though much simplified, still has disadvantages. One disadvantage is that variances of $m$ and $r$ estimated from equation 7 are asymptotic variances. We cannot directly use these variances to calculate their confidence intervals due to the non-zero covariance between these two parameters. Therefore, we have to adopt other methods such as the bootstrap to construct their confidence intervals. It also assumes that changes in the frequencies of inoculants are due exclusively to recombination and immigration. Selection, drift, or both might also significantly affect the estimation of $m$ and $r$.

The model can be simplified further by considering only the novel isolates in the mixed population as follows. Assume that DNA fingerprints (independent of the RFLP data used in the likelihood procedure) are sufficient to differentiate the inoculated isolates from the noninoculated (= novel) isolates. The genotype frequencies in the parental and immigrant populations can be estimated directly by using the allele data obtained in the first collection of inoculated and uninoculated plots. If we assume that all of the novel isolates in the second sample (the mixed population) originated from immigration or recombination, then the proportion of novel isolates originating from immigration is $m$ and the proportion from recombination is $1-m$. If the proportion of asexual progeny is known (that is, the asexual progeny can be identified unequivocally with DNA fingerprints), then the expected probability of genotype $g_{i}$ in the novel isolates of the mixed population will be

$$
p_{g_{i}}=p_{M} p_{g / M}+p_{R} p_{g / R}=m M_{g_{i}}+(1-m) R_{g_{i}}
$$

The likelihood of genotype $g_{i}$ with probability $p_{g_{i}}$ being observed $n_{i}$ times among the novel isolates of the mixed population is

$$
L(m)=\frac{N !}{n_{1} ! n_{2} ! \ldots n_{i} !} \prod_{i}\left[m M_{g_{i}}+(1-m) R_{g_{i}}\right]^{n_{i}}
$$

and the log-likelihood is

$$
\ln L(m)=c+\sum_{i} n_{i} \ln \left[m M_{g_{i}}+(1-m) R_{g_{i}}\right]
$$

After partial differentiation

$$
\frac{\partial \ln L(m)}{\partial m}=\sum_{i} \frac{n_{i}\left(M_{g_{i}}-R_{g_{i}}\right)}{m M_{g_{i}}+(1-m) R_{g_{i}}}
$$

$m$ can be estimated by setting equation 12 to zero and solving it. The large-sample variance of $m$ is

$$
\operatorname{Var}(\hat{m})=E[I(m)]^{-1}=\left\{-\sum_{1}^{i} \frac{n_{i}\left(M_{g_{i}}-R_{g_{i}}\right)^{2}}{\left[m M_{g_{i}}+(1-m) R_{g_{i}}\right]^{2}}\right\}^{-1}
$$

The variance estimated with equation 13 is the asymptotic minimum value (4).

The hypothesis that the estimated $m$ differs from an arbitrary value of $m\left(m_{0}\right)$ can be tested by using the following formula (7).

$$
\chi^{2} \approx 2\left[\ln L(\hat{m})-\ln L\left(m_{0}\right)\right]
$$

As previously, the chi-squared value can be calculated by substituting into equation 11 the estimated value of $m$ and the hypothesized value for $m$. This chi-squared test has one degree of freedom.

One drawback of using MLEs is that there are a large number of terms in the sum. But the resulting complex equation can be solved with a computer program or through iterative procedures (9). Another drawback of MLE is that the parameter(s) estimated might result in a negative $m$ or an $m$ larger than 1, which has no biological meaning. If MLE approaches give biologically uninformative results, other statistical methods might be required.

One alternative non-MLE method is to use a modification of the method we described previously (11). As stated in our original paper and reinforced by Brown (1), the accuracy of the estimates will depend on the difference in allele frequencies between parental and immigrant populations. If differences in allele frequencies between the source of immigrants and the source of recombinants are not extreme, then our original method can be modified to utilize all novel isolates in the mixed population. The relative proportions contributed by recombination and immigration can be estimated using an interative procedure with the following formula

$$
\hat{m}=\sum_{i} \frac{n_{i}}{N}\left[\frac{m M_{g_{i}}}{m M_{g_{i}}+(1-m) R_{g_{i}}}\right]
$$

in which $N$ is the sample size. Substitute $m$ with a series of values between 0 and 1 until $\hat{m}$ approximately equals $m$. Standard errors can be estimated using bootstrapping.

Note that equation 15 can be derived from equation 12 by multiplying the latter with the term $m(1-m)$. This equation always has two solutions, i.e., $m=0$ and $m=1$. Users of this formula have to determine if there is a solution for $0<m<1$. The $m$ esti- 
mated from equation 15 is equivalent to that estimated from equation 12 as long as $m$ falls between 0 and 1 .

When the differences between the two populations are large (ideally with different fixed alleles for each locus in parental and immigrant populations), most of the isolates will fall into the two extreme categories and our original method should give estimates for recombinants and immigrants that are close to the true values. If the genetic markers can distinguish unequivocally among asexual, sexual, and immigrant progenies (a suggestion of Brown [1]), then the statistical analysis is simple. The relative contributions to the genetic structure of the population are found by dividing the observed number of immigrant, recombinant, and asexual progeny by the total number of observations. This is the intuitive result developed by Brown (1) in his equations 6,7 , and 8 . And the estimation of standard errors on those frequencies by bootstrapping is straightforward.

We reanalyzed our original data with the methods shown in equations 12 and 15. The results are shown in Table 1. Results from this analysis show that our original method underestimated immigration rates by approximately $10 \%$ for both the mid- and late-season collections. Using equation 14 , we found that the differences between the original and new estimates of immigration were not statistically significant for either collection $\left(\chi^{2}=3.50\right.$ and 2.18 for the mid- and late-season collections, respectively).

Brown raised a relevant concern (aspect ii in literature citation 1) when he noted that all possible pairwise crosses would not occur among the potential parents for fungal pathogens with a heterothallic mating system. In our original data set, we noted that the inoculated isolates did not appear to contribute equal numbers of recombinants, and this partially explained the significant disequilibrium found among RFLP loci in the novel isolates (Table 7 in literature citation 11). We did not factor the heterothallic mating system into our original calculation of $m$ and $r$, and the likelihood methods described here do not specifically account for heterothallic mating systems. Though we agree that a heterothallic mating system is likely to influence the estimates of $m$ and $r$, we believe that the error will be mainly random unless different alleles are fixed in the immigrant and parental populations. Under this scenario (different fixed alleles), recombinants and immigrants can be differentiated based on their multilocus genotypes.

Brown's third point (aspect iii in literature citation 1) was that an ideal experiment would utilize inoculants that differ from potential immigrants by fixed differences at approximately nine loci. His fifth point (aspect $v$ in literature citation 1) was that at least 500 isolates should be sampled from each population at each point in time. We agree that this would be an ideal experimental design, though it may be more difficult to conduct this experiment than is anticipated. In our international collection of over 2,000 characterized strains of $M$. graminicola, we found many isolates that possessed one or two rare alleles across the 11 RFLP loci that we surveyed (6). But it was very unusual to find an isolate with rare alleles at more than three loci, and no isolates possessed rare alleles at more than five loci. Choosing inoculant isolates that differ from potential immigrants by fixed differences at nine loci may prove especially challenging for fungi that exhibit moderate to high de-

TABLE 1. The estimated proportion of immigrants among the novel isolates of Mycosphaerella graminicola in mid- and late-season collections from a field experiment (11)

\begin{tabular}{lccc}
\hline & \multicolumn{3}{c}{ Immigrants $^{\mathrm{a}}(\%)$} \\
\cline { 2 - 4 } Populations & Method 1 $^{\mathrm{b}}$ & Method 2 $^{\mathrm{c}}$ & Method 3 $^{\mathrm{d}}$ \\
\hline Mid season & 80.0 & $90.4 \pm 9.5^{\mathrm{e}}$ & 90.4 \\
Late season & 30.4 & $38.8 \pm 9.1$ & 38.8 \\
\hline a The proportion of recombinants is 1 minus the proportion of immigrants. \\
b Based on the method described by Zhan et al. (11). \\
c Based on equation 12 (maximum likelihood). \\
d Based on equation 15 (posterior probabilities). \\
e 95\% confidence intervals are identical for recombinants and immigrants.
\end{tabular}

grees of gene flow. We found it more effective to use an independent type of genetic marker (a DNA fingerprint) to differentiate inoculants from recombinants and immigrants.

Large sample sizes certainly increase confidence that the interpretations drawn from a data set are correct. And we agree that sample sizes should be as large as possible given the normal constraints in funding and time allocated to complete an experiment. It is clear that our sample of only 45 isolates from the uninoculated plots was low, and we agree that a larger sample of isolates should be drawn from the population of potential immigrants. However, we believe this small sample size did not materially affect our results in this instance. We still conclude that the relative importance of immigration and recombination to the genetic structure of field populations of $M$. graminicola shifted over the course of the growing season (11). The $M$. graminicola population existing at the Oregon State University Plant Pathology experiment station may be the most intensively characterized population of any plant pathogen. We base this statement on our analysis of 10 to 13 nuclear RFLP loci, two DNA fingerprints, and mitochondrial haplotypes in over 2,000 isolates sampled from this site over a 6-year period. The allele frequencies in the collection of 45 isolates made as part of this experiment in 1995 did not differ significantly from the frequencies in our collection of 711 isolates made in $1990(2,3,10)$.

We agree with Brown that it is important to develop appropriate statistical methods that are as powerful as the molecular tools now used to study the population genetics of plant pathogens. And we hope that the maximum likelihood methods that we developed in our reply will find widespread use among plant pathologists and other biologists trying to differentiate among the contributions of immigration, recombination, and asexual reproduction to the genetic structure of pathogen populations.

\section{ACKNOWLEDGMENTS}

We thank H. R. Kuensch and H. R. Roth, Department of Mathematics, ETH-Zurich, for assistance with developing maximum likelihood estimates and for clarifying the presentation of our formulas. We also thank J. Taylor, Department of Animal Science, Texas A\&M University, for contributing significantly to developing the formulas used to estimate variances based on the maximum likelihood estimates.

\section{LITERATURE CITED}

1. Brown, J. K. M. 2000. Estimation of rates of recombination and migration in populations of plant pathogens. Phytopathology 90:320-323.

2. Chen, R. S., Boeger, J. M., and McDonald, B. A. 1994. Genetic stability in a population of a plant pathogenic fungus over time. Mol. Ecol. 3:209-218.

3. Chen, R. S., and McDonald, B. A. 1996. Sexual reproduction plays a major role in the genetic structure of populations of the fungus $M y$ cosphaerella graminicola. Genetics 142:1119-1127.

4. Edwards, L. W. F. 1992. Likelihood: Expanded Edition. The Johns Hopkins University Press, Baltimore.

5. McDonald, B. A., Mundt, C. C., and Chen, R. S. 1996. The role of selection on the genetic structure of pathogen populations: Evidence from field experiments with Mycosphaerella graminicola on wheat. Euphytica 92:73-80.

6. McDonald, B. A., Zhan, J., Yarden, O., Hogan, K., Garton, J., and Pettway, R. E. 1999. The population genetics of Mycosphaerella graminicola and Phaeosphaeria nodorum. Pages 44-69 in: Septoria on Cereals: A Study of Pathosystems. J. A. Lucas, P. Bowyer, and H. M. Anderson, eds. CAB International, Wallingford, United Kingdom.

7. Rice, J. A. 1995. Mathematical Statistics and Data Analysis. 2nd ed. Duxbury Press, Belmont, CA.

8. Shenton, L. R., and Bowman, K. O. 1977. Maximum likelihood estimation in small samples. Charles Griffin and Company Ltd., London.

9. Weir, B. S. 1996. Genetic Data Analysis II. Sinauer Associates Inc., Sunderland, MA.

10. Zhan, J. 1998. The role of immigration, mating system and selection on the genetic structure of populations of the wheat pathogen Mycosphaerella graminicola. Ph.D. dissertation. Texas A\&M University, College Station.

11. Zhan, J., Mundt, C. C., and McDonald, B. A. 1998. Measuring immigration and sexual reproduction in field populations of Mycosphaerella graminicola. Phytopathology 88:1330-1337. 